very special circumstances, excluded this affection from treatment, because of the difficulty of eliminating in an out-patient department that class of phthisical patients who, being already the subject of auto-inoculations, cannot, it seems to me, except under very special precautions, safely be treated by the method of inoculation.

Lupus. - If we except one of our very earlfest cases-where the results of a few weeks' treatment were, probably owing to the administration of too large doses of the vaccine, such as to discourage the patient, and to lead him to abandion the treatment-we may say that the inoculation treatment has in all the cases of lupus we have dealt with ameliorated, but so far only in one case cured, the disease. Not infrequently we have seen certain of the patches completely cured, while the disease in other regions has remained refractory. These only partially successful results, which contrast in a striking manner with those obtained in connexion with tubercular ulceration affecting the deeper tissues, depend, it seems to me, not so much upon a defective power of response to inoculations on the part of the lupus patient, as upon the inadequate manner in which the antibacterial substances come into application upon the tubercle bacilli in the case where these are disposed in a skin which is but poorly supplied with blood. I do not doubt that if it were possible to superadd to the treatment by inoculation another form of treatment which achieves, as the Finsen light appears to do, a proper transudation of lymph into the skin, the efficiency of the inoculation treatment as applied to lupus skin, the efficiency of the inoculation treatment as

Tubercular ulceration of subcutaneous tissues and bone. - The clearest and most unfallacious evidence of the advantage which can be clearest and most unfallacious evidence of the advantage which can be derived from the therapeutic inoculation of tubercle vaccine can be furnished in connexion with tubercular ulceration of the subcutaneous tissues. It is not a question here of the achievement of success in a certain percentage of cases where ordinarv surgical methods have failed. Up to the present, at any rate, it has been a question of uniform success. The following series of cases, all of which, except the last, are available for inspection, furnish evidence of what can be achieved by and which might quite well have been reckoned desperate.

[Professor Wright here read particulars of three interesting cases in proof of his contention.]

Tubercular invasion of lymphatic glands.--Next perhaps to tuber rular ulceration of the subcutaneous tissue tubercular affections of the lymphatic glands furnish the clearest evidence of the efficacy of therapeutic inoculation of tubercle vaccine. This result, as reflection will show, is in accordance with what might have been expected a priori in view of the fact that the tubercle bacilli are here disposed right in the path of the lymph stream which is passing back through the gland to the blood. I do not myself doubt from what I have seen of the effect of inoculation on tutereulous glands that the extirpation of these by surgical methods, as well as the purely climatic treatment of this affection, are destined to give place to the therapeutic exploitation of tuberculin inoculations controlled by the determination of the opsonic index, and combincd with hot sand poultices and rubefacients, or other measures which like these will produce an ampler lymph flow in the whole territory-or may I call it water-shed or collecting basin--whose lym

[Professor Wright here read brief notes relating to results achieved in complicated or practically uncomplicated cases of tuberculous glands.]

Tubercular disease of the genito-urinary system - From some points of view more convineing, in others only less convincing, than the results obtained in connexion with lesions which are directly accessible to sight and touch, are the results obtained in connexion with tubercular disease of the fgenito-urinary system, in particular in the cases where these involve the bladder. We have in the fact that these cases are associated with distressing pain and frequency, and in the fact that the presence or absence of tubercle bacilli in the urine can here be determined by microscopic observation, the means of measuring success and failure.

[Professor Wright here gave a brief abstract of certain cases which have been under treatment.

In view of the very favourable and, what is almost more important, uniformly successful results which can, as will have appeared, be obtained even in the most intractable cases of localised tubercular infection by the therapeutic inoculation of tuberculin carried out under the safeguards explained above; and in view of the fact that not less favourable results can be obtained in connexion with the treatment of other localised infections by the aid of the corresponding bacterial vaccines; I do not hesitate to contend that we have in the power of raising the antibacterial power of the blood with respect to any invading microbe, out of all comparison the most valuable asset in medicine. I would, in view of this new asset in medicine, fain induce the surgeon to abate something from his conviction that extirpation and the application of antiseptics offer in connexion with bacterial infection the only possible means of cure; I would have the surgeon resort to extirpation only when the phy. sician tells him that all other means have been exhausted and I would have the physician assume everywhere the rôle of an immunisator; and I would have him defer handing his patients over to the surgeon before he has tried in every case of localised bacterial infection which is unassociated with immediate risk to life the therapeutic inoculation of the appropriate bacterial vaccine.

(To e concluded.)

\section{THE TREATMENT OF TUBERCULOSIS BY} TUBERCULIN.'

By WILliam BULLOCH, M.D. ABerd., BACTERIOLOGIST TO THE LONDON HOSPITAL.

THE main currents of intellectual activity in the domain of pathology have flowed for the past 30 years towards the elucidation of the causes of disease. We have passed away from the earlier symptomatic and anatomical and have arrived at etiological concepts. A most important advance has been the demonstration that a number of diseases owe their origin to the invasion of the body by parasitic microbes. The doctrine that these microbes are specific has triumphed over other conceptions and may be said to be a universal belief at the present time. The aim of this absorbing and intricate study has been the construction of a scientific theory of disease and depending on this the elaboration of a rational therapy. But even this rational therapy is gradually narrowing itself down to a specific therapy. Remedies are being sought which lead to the exclusive destruction of the particular specific microbe which has been proved to be the cause of the given infection. Following Edward Jenner, the pioneers of bacteriology, notably Pasteur, sought to induce a prophylaxis by the inoculation, mostly in attenuated form, of the etiological agents themselves, or products derived from them. To Koch, however, belongs the credit of having been the first to attempt to cure an infection by a specific remedy-viz., tuberculin in tuberculosis. When we reflect for a moment on the problem which confronted Koch it will be manifest that the results of such treatment cannot be compared with those in which a prophylaxis is induced against an infection like variola, where, in the natural course of events, an extraordinary degree of immunity is established for a number of years, or for the life of the individual.

The extensive study of tuberculosis has shown that man and beast are infected to an alarming extent. In man, at any rate, the observations of Naegeli and others lead one to assume that the disposition is almost universal. If exposed to tubercle bacilli for any prolonged period few escape without some lesion. By a long acclimatisation the tubercle bacillus has become extraordinarily parasitic and seemingly incapable of multiplying its numbers outside the living body. The parasitism is also evinced in the extreme chronicity of the tuberculous processes and the fact that the bacillus is not comparable in the intensity of its pathogenic effects with the rapidly fatal septicæmic blood infections. Recently, Theobald Smith has developed the idea, paradoxical as it may seem, that the more habituated a microbe becomes to subsisting in the living tissues-i.e., the more parasitic it becomes-the less actively aggressive it is and the more it endeavours by pure methods of defence to secure a nidus wherein it may multiply undisturbed. The throwing up of cellular works in the shape of a specific granuloma by the host may thus be a disadvantage in so far that the bacillus is protected in an abode where it may sojourn until opportunity occurs for transmission to another host. It is, however, generally supposed, arguing from the analogy of lifeless irritamenta, that the inclosure of the alien substance by a wall of granulation tissue is a sign that the organism is endearouring to cope with the offensive agent by excluding it.

The most recent studies in immunity to tubercle lead us, however, to conclude that the inclosure of the tubercle bacillus by a wall of poorly vascularised granulation tissue is a drawback, as the antibacterial forces which we know to exist in the body humors are kept at bay, the parasite remaining locally, and being at long intervals discharged outward. In general the defence offered by the bacillus is so powerful that only a relatively small degree of immunity is developed by the host. This statement rests on a large body of evidence and it cannot ke expected, even from a remedy like tuberculin, that a high degree of immunity can be attained in a short space of time or that the curative

1 A paper read before the Royal Medical and Chirurgical Society on Nov. 28th, 1905. 
effect of this remedy is comparable to its prophylactic effect. We cannot evade the conclusions reached by a generation of medical scientists, and it seems to me that this is all the more important at the present time, when the eyes of the civilised world scan the scientific horizon with anxiety for the long-expected " cure for consumption."

The foundation of Koch's work-to my way of thinking his greatest work-was the observation that even small quantities of killed suspensions of tubercle bacilli can produce death in animals already tuberculous. By reducing the dose to very minute proportions he found in a certain number of cases that the disease can be brought to a standstill. He found, however, that the dead bacilli which he had injected remain locally for long periods
and may ultimately give rise to abscesses. Whatever amelioration of the tuberculous disease had taken place must have been due to substances which passed out of the bodies of the dead bacilli injected and this gave the suggestion that such substances might be prepared artificially and injected in a form capable of rapid absorption. In this way originated tuberculin, now described as "old tuberculin" (T.O.), a concentrated glycerine extract of tubercle bacilli. When this extract was injected into tuberculous individuals the manifestations witnessed were pyrogenic and phlogogenic, the latter consisting of inflammation, leucocytic emigration, and necrosis of tuberculous foci, which were visible to the eye. Koch regarded this local necrotising action as an essential element in the cure, and the explanation of the action accepted by him was that originated by Babes and spoken of as the "addition theory," which assumed that the tubercle bacillus produces certain substances, some of which bring about a coagulation necrosis. This necrosis acts deleteriously on the bacillus and may cause its death. By the inoculation of tuberculin the necrotising effects are increased in intensity-a condition which seriously affects the vitality of the bacillus, so that ultimately a phase of tranquillity, or even cure, is established in the infected individual. The rise of temperature which accompanies the local reaction was looked upon as specific, in so far as it occurred only in tuberculous individuals. In recent years it has been shown, especially by Preisich and Heim, that the tuberculin reaction is more complicated than was originally believed. They have proved that the presence of tuberculous tissue is not necessary for the onset of the tuberculin febrile action. By transplanting collodion sacs containing the bacilli alone into the peritoneal cavity of guinea-pigs, followed by the inoculation of suitable doses of tuberculin, they have obtained typical tuberculin reactions. This reaction must be due either to substances which have diffused out of the collodion capsules, and which have united with the tuberculin to give rise to a substance which produces the reaction, or in virtue of the toxic action of the tuberculin a change is induced in the cells which renders them capable of manifesting the tuberculous reaction. Preisich and Heim have, in fact, shown that there are substances in the serum of tuberculous animals which, on admixture with tuberculin, are capable of setting up a typical reaction in normal animals. As they found, however, that such substances may be present in normal serum they concluded that the rise of temperature following an injection of tuberculin cannot be looked upon as specific of tuberculosis. The tuberculin reaction is, however, generally regarded as specific. Whatever may be the ultimate views held on the nature of this tuberculin reaction there is no doubt that 15 years ago Koch and other investigators considered that a local reaction is a necessary part of the curative action of tuberculin.

No remedy in therapeutios has suffered the fate of Koch's tuberculin. At first hailed with ecstasy as the long-sought panacea for tuberculosis, its short-lived glory was succeeded by a period of absolute rejection and then one of indifference, so that to-day it may be regarded as having dropped out of the pharmacopcia of even the serious medical practitioner. So convinced, however, was Koch of the virtues of tuberculin that he proceeded to perfect his discovery, and as he had observed that with the repeated inoculation of this substance a period of tolerance is established when the reaction, and with it, according to him, the curative properties come to an end, he strove to prolong the reaction period and achieved this to a certain extent by tuberculin $A$, an extract of the tubercle cultures with $\frac{N}{10}$ sodium hydrate solution. 1 The disadvantage of tuberculin $\mathbf{A}$ was, however, that abscesses were frequently produced, as the bacillus remained unabsorbed for long periods at the point where it had been inoculated. This led Koch to the idea that the difficulties of absorbability might be got over by the mechanical comminution of the bacillus. To this end highly virulent bacilli were dried in vacuo and then comminuted by machinery. The dust was treated with distilled water and the mixture was then placed in a centrifuge rotating 4000 turns per minute. In this way an opalescent fluid (T.O.) and a deposit were obtained. The deposit was then worked up with successive quantities of water and was named T.R. This, under the name of tuberculin T.R., or Koch's new tuberculin, is sold in commerce in bottles containing ten milligrammes solid substance per cubic centimetre. The opalescent fluid (T.O.) possesses properties analogous to the old tuberculin, whereas the T.R., as Koch himself recognised, acts independently of a local reaction which, in fact, should be prevented at all costs. Koch, however, recommended forcing the inoculations as far as was compatible with the absorption of the tuberculin.

Following on the work of Koch other bacillary products have been recommended, such as tuberculoplasmin (Buchner), tuberculocidin and antiphthisin (Klebs), oxytuberculin (Hirschfelder), tuberculin (Denys), tuberkulol (Landmann) and tuberculin (Beraneck). These remedies have been vaunted as superior, whereas few, if any, attain even the measure of success of Koch's tuberculin. Taking them as a group we may apply the term tuberculin as a collective name for soluble or emulsionised products prepared from the tubercle bacillus. Between the years 1890 and 1897, while Koch was investigating the therapeutic value of his tuberculins, a remarkable event in medicine had interposed itself. I refer to Behring's discovery and application of serum therapy. At once it was thought that at last here was the line of real advance in the treatment of tuberculosis, but, despite the most strenuous effort, nothing even worthy of comment has been achieved by the various antituberculous sera which have been manufactured, and the attempts and methods have been so manifold that one is inclined to prophesy that the era of tuberculous sero-therapy is past. What has been the result of eight years' experience with Koch's new tuberculin? Already, within a year of its introduction, a large number of authors here and abroad had written commentaries of disappointment. Others have condemned it as being contaminated or even infected with the tubercle bacillus itself Others, again, who have given it a serious trial report that the results depend not so much in the remedy itself as in the manner of its exhibition. Authorities like Turban, Goetsch, Petruschky, Spengler, and Moeller have pronounced in its favour, even in pulmonary tubercle. With few exceptions, dermatologists do not seem to have been convinced of its efficacy. The work of a man like Koch cannot be brushed aside as fanciful, and there is, I think, no doubt at all that tuberculin is a substance which possesses remarkable curative powers in tuberculosis Recently in this country Wright has opened up the question of tuberculin therapy and has achieved memorable results in the treatment of the localised forms of tubercle. He has shown that protective substances of opsonic nature exist in the normal serum and that the opsonic content of the serum can be increased by the inoculation of tuberculin. It is manifest from his work that the chief defensive mechanism against tubercle exists in the serum and not in the cells, as was believed, and the destruction of the bacilli by the cells must be preceded by the action of the serum. He has also elaborated a method by which it is possible to determine the opsonic content of the serum and he has utilised this determination as a guide to the inoculation of the infected individual with tuberculin. An important outcome of his studies has been the demonstration that tuberculin is an extremely potent agent and that its dosage hitherto has been excessive. I have worked a good deal with the methods invented by Wright and propose to give some of the details of this work in connexion with the treatment of tubercle, especially in its localised forms.

In his earlier work Wright noted that the opsonic index of individuals suffering from tubercle is lower than that of normal people. In order to determine whether this is constant I have examined first of all the indices of 84 healthy people, comprising 44 medical students and 40 hospital nurses. In all cases the serum was compared with my 
own, which was regarded as unity. The result was as follows :-

Opsonic index of 44 healthy medical stud mnts compared with the serum of the writer $=1 \cdot 0$

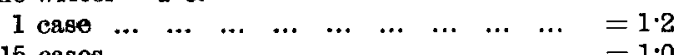

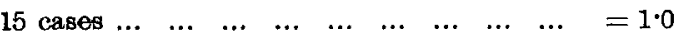

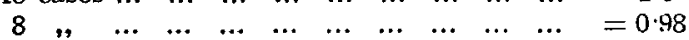

$$
\begin{aligned}
& \begin{array}{llllllllllll}
8 & \cdots & \ldots & \ldots & \ldots & \ldots & \ldots & \ldots & \ldots & \ldots & \ldots & =0.98 \\
7 & \ldots & \ldots & \ldots & \ldots & \ldots & \ldots & \ldots & \ldots & \ldots & \ldots & =0.97
\end{array} \\
& \begin{array}{llllllllllll}
7 & \cdots & \ldots & \ldots & \ldots & \ldots & \ldots & \ldots & \ldots & \ldots & \ldots & =0.97 \\
2 & , & \ldots & \ldots & \ldots & \ldots & \ldots & \ldots & \ldots & \ldots & \ldots & =0.96
\end{array}
\end{aligned}
$$

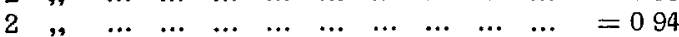

$$
\begin{aligned}
& 6 \%
\end{aligned}
$$

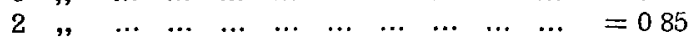

$$
\begin{aligned}
& \begin{array}{llllllllllll}
1 \text { case } & \ldots & \ldots & \ldots & \ldots & \ldots & \ldots & \ldots & \ldots & \ldots & =0.80
\end{array}
\end{aligned}
$$

Opsonic index of 40 healthy hospital nurses-

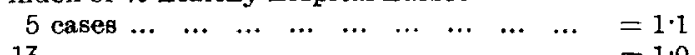

$$
\begin{aligned}
& \begin{array}{llllllllllll}
13 & , & \ldots & \ldots & \ldots & \ldots & \ldots & \ldots & \ldots & \ldots & \ldots & =1 \cdot 0
\end{array} \\
& \begin{array}{llllllllllll}
5 & , & \ldots & \ldots & \ldots & \ldots & \ldots & \ldots & \ldots & \ldots & \ldots & =0.98
\end{array}
\end{aligned}
$$

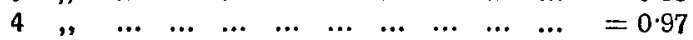

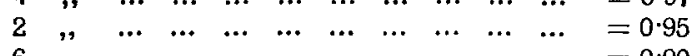

$$
\begin{aligned}
& \begin{array}{llllllllllll}
6 & , & \ldots & \ldots & \ldots & \ldots & \ldots & \ldots & \ldots & \ldots & \ldots & =0.90
\end{array}
\end{aligned}
$$

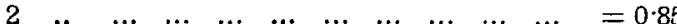

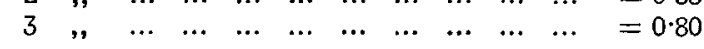

Taking the two series together we get an average index of 0.96 for the 84 people, the variations in health ranging from 0.8 to 1.2 . Coming to cases of tuberculosis I have already published in the Transactions of the Pathological Society the determination of the opsonic indices of 150 cases of lupus occurring in the Skin Department at the London Hospital. . The great majority of these cases attended the hospital for the purpose of $x$ ray or Finsen light treatment. Compared with the average opsonic index of 0.96 obtained in normal people the average for the 150 cases of lupus was $0 \cdot 75$, the cases being distributed as follows :-

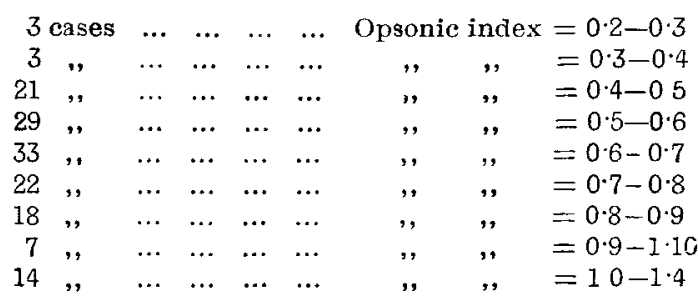

75 per cent. of the lupus cases showed an opsonic index below the lowest normal limit of $0 \cdot 8$.

Clinical data were available whereby the results obtained by Finsen therapy could be compared with the height of the opsonic index, and on analysing these data it was found in general that where the opsonic index is well below the lowest normal limit the results of Finsen therapy have been disappointing, whereas with indices in the normal limits or above them the clinical impression has been that the cases have done well. The mode of action of the exposure to light in Finsen's method has been the subject of much inquiry. It is admitted on all hands that under certain conditions of experiment light acts as a bactericidal agent. This, however, has not been proved in the case of Finsen light, where the rays have to traverse the skin. In all probability, and the above results lend support to this view, Finsen light acts mainly by producing a local inflammation with great vaso-dilatation and a transudation of large quantities of plasma in the areas exposed to its action. If the serum is poor in antibodies the results of Finsen therapy are disappointing and this suggests that its value might be enhanced if the antitropic content of the serum were increased by previous inoculations of tuberculin. 11 cases of surgical tubercle were examined with the following result :-

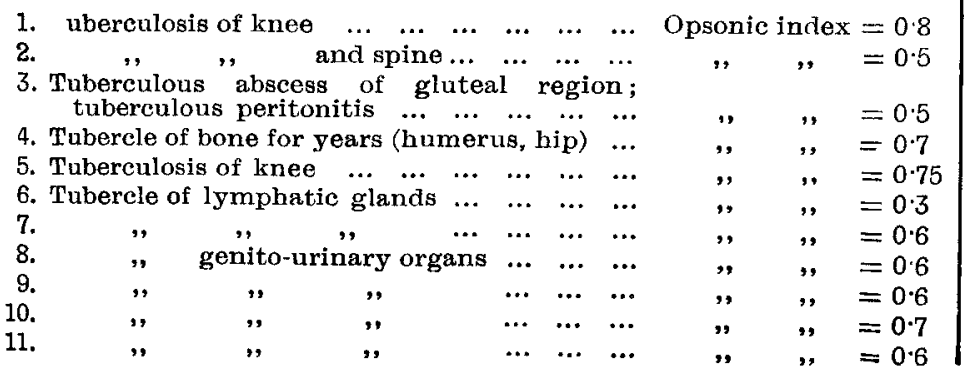

\begin{tabular}{|c|c|c|c|c|c|c|c|c|c|}
\hline Case & 1 & $\ldots$ & $\ldots$ & $\ldots$ & $\ldots$ & $\ldots$ & Opson & index & $=0.7$ \\
\hline " & 2 & $\ldots$ & .. & $\ldots$ & $\ldots$ & $\ldots$ & ," & ," & $=0.6$ \\
\hline ", & 3 & $\ldots$ & ... & $\ldots$ & $\ldots$ & $\ldots$ & " & ", & $=0.7$ \\
\hline ", & 4 & $\ldots$ & ... & $\ldots$ & $\ldots$ & $\cdots$ & " & ", & $=0.6$ \\
\hline , & 5 & $\ldots$ & ... & $\ldots$ & $\ldots$ & $\cdots$ & to & ", & $=0.5$ \\
\hline , & 6 & $\ldots$ & $\ldots$ & $\ldots$ & $\ldots$ & $\cdots$ & " & , & $=0.4$ \\
\hline ", & 7 & $\ldots$ & $\cdots$ & ... & ... & $\cdots$ & $"$ & $"$ & $=0.6$ \\
\hline ", & 8 & $\cdots$ & $\cdots$ & $\cdots$ & $\cdots$ & ... & ", & " & $=0.65$ \\
\hline ", & 9 & $\cdots$ & $\cdots$ & $\cdots$ & $\cdots$ & $\cdots$ & ", & , , & $=0.75$ \\
\hline " & 10 & $\ldots$ & $\cdots$ & $\ldots$ & $\ldots$ & $\cdots$ & " & , & $=0.6$ \\
\hline ", & 11 & $\ldots$ & $\cdots$ & $\ldots$ & $\ldots$ & $\cdots$ & ," & ," & $=0.5$ \\
\hline ", & 12 & $\ldots$ & $\cdots$ & $\cdots$ & $\cdots$ & $\cdots$ & , & , , & $=0.45$ \\
\hline " & 13 & $\ldots$ & $\cdots$ & $\ldots$ & $\ldots$ & $\ldots$ & 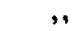 & ," & $=0.6$ \\
\hline ," & 14 & $\ldots$ & $\ldots$ & $\ldots$ & $\ldots$ & $\ldots$ & ," & ., & $=0.86$ \\
\hline
\end{tabular}

14 cases of so-called " sanatorium cures" or arrested cases of pulmonary tuberculosis were examined and the index was found depressed in all except one. These cases were all from the well-to-do classes and in at least one of these cases, which was considered a perfect cure, the disease has relapsed within a year.

From these determinations in healthy and diseased people it would appear that an index below 0.8 is abnormal. It is not possible to say when a low index is encountered whether the individual is already infected or is merely likely to be so. The very early diagnosis of tubercle is difficult or impossible by ordinary clinical methods. Arguing from the case of staphylococcus lesions which are visible in the skin the probability is that a very low index indicates that infection has occurred. An abnormally high index is probably a sign of infection but cannot be used as a prognostic, as it may occur in those cases which do well or in those which are quite hopeless. Repeated determinations of the fluctuations in the index are probably of more importance in forming an opinion on the future of the case.

The exact nature of the opsonic action has not yet been worked out. All investigators, however, are agreed that where different bloods are compared the variable factor is the serum and not the leucocytes. The existence of Metchnikoff's hypothetical "stimulins "-bodies which stimulate the leucocytes-still lacks demonstration. The relation of the leucocytosis, which accompanies most infections, to the opsonic content of the serum has not been made out. Bulloch and Ledingham in a large number of experiments failed to demonstrate that there is any close relationship between these factors. With certain drugs, such as sodium cinnamplicum ("hetol") and tallianine, which produce marked leucocytosis, no increase in the quantity of the opsonin could be determined. Subcutaneous inoculations of a solution of nuclein from yeast had, however, a very marked effect in producing an opsonic increase, although the leucocytosis was inconstant.

Specificity of opsonins. - This question has hitherto not been touched in the various memoirs which have appeared on opsonins. In conjunction with my assistant, Dr. G. T. Western, I have recently been able to demonstrate that a high degree of specificity exists. This has been shown in two classes of experiments. In the first the opsonic power of a serum was tested against both staphylococcus albus and tubercle bacillus. The serum was then mixed with one or other of these microbes, and after a sojourn in the incubator the mixture was subjected to the prolonged action of the centrifuge, whereby the microbe was thrown down as a deposit, the supernatant liquid being then tested for both microbes. The result of experiments of this type is that the supernatant liquid is deprived of its opsonin for the particular microbe with which it has been in contact while it largely retains its opsonin for the microbe with which it has not been digested. In the second class of experiment the serum of human beings was tested repeatedly, both against tubercle bacillus and against staphylococcus. Injections of tuberculin produced an increase in the tuberculous opsonin while leaving the quantity of staphylococcic opsonin unaltered, and vice vers $\hat{\alpha}$. We may thus assume the demonstrtion of the specific nature of the opsonins in the serum.

Iffects of the inoculation of tuberculin.-In almost every instance of scores of inoculations I have observed the occurrence of a negative phase. In the cases where this has been absent it has followed a very minute dose or the same dose had been injected several times previously. The determination of opsonic curves also leads me to believe in the correctness of Wright's view that the negative phase is one of importance. It is extraordinarily variable, even where the same doses of tuberculin are given to different. people. In some cases the descent is abrupt and steep, in 
other cases the negative phase persists over a number of days, and frequently the subjective symptoms of discomfort on the part of the patient are increased. I have on several occasions seen a definite exacerbation of the symptoms associated with a prolonged negative phase. The maintenance of Wright's "high-tide" phase of the immunity curve is subject to great variations. In most cases, as far as my experience goes, the " high tide" is in the course of a few days succeeted by a fall again, although the fall is rarely as deep as that shown prior to the injection of tuberculin. In spite of this the improvement may continue, a fact which leads me to assume that there are other factors at work which at the present time cannot be measured. The vaccine employed was Koch's new tuberculin, as manufactured by Meister, Lucius, and Brüning, at Höchst.

\section{CAses.}

All the cases which I have had to treat have been severe. The majority of them, in fact, had passed from the hands of physicians and surgeons and were regarded as incurable. It is impossible to speak with certainty as to cure, because a great deal depends on what is meant by this term. In ordinary medical parlance there is a time element in the question and one must distinguish between a temporary and a permanent cure. Thus one of the patients I have treated was a boy the subject of tuberculous glands of the neck. An extensive operation was undertaken and the diseased glands were removed from both sides of the neck. After a residence of some four weeks in the hospital he was dismissed " cured," although there was a slight discharge from one of the wounds. But this discharge never stopped and six months later he was as bad as ever with two sinuses leading down to softening glands. By the use of tuberculin alone these glands have disappeared and the wound is completely closed, so that there is a temporary cure; but no one can say that the cure is permanent until the individual has been a long time under observation. I have treated two cases of genito-urinary tuberculosis, seven cases of lupus, and two cases of gland tubercle. The results may be briefly stated as follows, substituting the letters of the alphabet for the patient's initials.

CASE 1.-A. B., by profession a surgeon. He suffered from genito-urinary tubercle which had been operated on on several occasions. The disease recurred and it was decided that further surgical interference was impossible. A prolonged course of tuberculin, with estimation of the opsonic indices, has led to immense improvement not only in the patient's general health but in the local area where the tuberculous deposits have disappeared. $\mathrm{He}$ is now in relatively good health.

CASE 2.-C. D. (16), a girl weighing 3 stones 4 pounds and the subject of extensive but quiescent tubercle of the spine and genito-urinary tuberculosis. Four months' treatment has caused great amelioration in the distressing symptoms to which she had been subjected. The patient is still under treatment.

CASE 3.-E. F. (23). Phthisis on the maternal side. The patient had pleurisy at the age of ten years. Lupus began in childhood and extended over the cheek and the eyelid. Treated with $x$ rays and 170 applications of Finsen light. Main area of disease healed up well but fresh nodules frequently made their appearance. His opsonic index on two occasions was 0.8 . Four inoculations of tuberculin

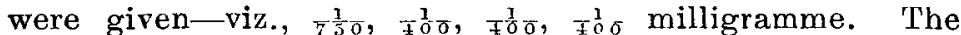
patient was discharged apparently cured. No recurrence in two months.

CASE 4.-G. H. (18), admitted 1901. History of lupus of three years' duration, disease affecting nose, cheek, mucous membrane of right nostril, soft palate, and uvula. Brsides $x$ rays, lactic acid, trichloracetic acid, and the Paquelin cautery, he had 225 applications of Finsen light. In August, 1905, there were still great thickening and odema of the lips; ulcerated patches on the lips and alæ nasi

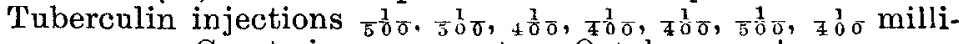
gramme. Great improvement. Ostober, œilema gone, ulcerated areas healing up. Still under treatment.

CASE 5.-I. J. (23). Lupus 20 years. Extensive area on the left cheek. Patient had 289 sittings of Finsen light and was apparently cured in 1904 ; relapsed repeatedly. Tuberculin inoculations begun August, 1905- $\frac{1}{50} \overline{0}$ 30 $3 \frac{1}{0}$ milligramme. Discharged apparently cured on Sept. 23rd, to be kept under observation. No recurrence, Nov. 21st.

CAsE 6.-K. L. (14). Lupus dates from 1901. Neck. cheek, and nose affected. Treatment: $x$ rays and 388 sittings of Finsen light. In July, 1905, several nodules still remained. Tuberculin inoculated in doses of $7 \frac{1}{5} \delta, 5 \frac{1}{50}, \frac{1}{4} \delta$, $\tau^{\frac{1}{0} \sigma}, \tau^{\frac{1}{0} \gamma}, \frac{1}{10}$ milligramme ; apparently cured; discharged Sept. 17th. No recurrence, Nov. 20th.

CAsE 7.-M. N. (22). Lupus 17 years, very extensive. Treated with Finsen light (540 applications) off and on for five years. Tuberculin inoculations have made her seedy and apparently cannot be tolerated.

CASE 8.-O. P. (26). Lupus of 11 years' standing. A most extensive case with great infiltration of the skin of cheeks, chin, neck, and chest. Treated by Finsen light 941 times. Excellent result achieved by this treatment, but disease relapsed. February, 1905, numerous nodules. Treated with tuberculin- $\frac{1}{7} \frac{1}{5}, \frac{1}{5} \overline{0}, 5 \frac{1}{50}, 3 \frac{1}{60}, \frac{1}{20}, 2 \frac{1}{0} \%$ milligramme. Apparently cured ; discharged August 20th. On Nov. 24th a few small nodules were detected.

CAsE 9.-Q. R. Lupus for 20 years, By 1902 it had spread over whole face and on to neck. In 1890 she was treated with old tuberculin with benefit but it is said to have upset her health. Prior to 1902 she was scraped 39 times. Since 1902 has had 1466 applications of Finsen light. In 1904 a finger was amputated for dactylitis. In April the opsonic index was 0.6 . An inoculation of $7 \frac{1}{50}$ milligramme of tuberculin raised her index to normal and it kept normal for three months. Since then she has had eight inoculations of $5 \frac{1}{0}, \frac{1}{40} \gamma, \frac{1}{40} \overline{0}, 3 \frac{1}{0} 0,5 \frac{1}{30},{ }_{3}^{\frac{1}{0}}, 2 \frac{1}{0} \overline{0}$, $\frac{1}{20}$ milligramme. Great improvement, although much scarred from scrapings.

CAsE 10.-S. T. (16). Tuberculous glands of neck on both sides. Excision of glands February, 1905. Recurrence. Sinus formation and softening of glands. Tuberculin treatment for two months. Apparently cured.

CAsE 11.-U. V. (10). Abdominal tuberculosis. Weight, 3 stones $2 \frac{1}{2}$ pounds. Treated with tuberculin without avail. Fæcal fistula for three weeks before death. P.M.-Lower part of abdomen was a cavity containing fæces. Extensive tuberculosis with matting of intestines.

I am indebted to Dr. Western for the results on most of the above-mentioned lupus patients and to Dr. J. H. Sequeira for permission to make use of these results.

Holland-road, W.

\section{A COMPARATIVE STUDY OF THE LINCOLN, MAIDSTONE, AND WORTHING EPIDEMICS OF TYPHOID FEVER. ${ }^{1}$}

By CHRISTOPHER CHILDS, M.A., M.D. OxoN., D.P.H., LECTURER IN THE DEPARTMENT OF HYGIENE AT LNIVERSITY COLLEGE, LONDON.

[IN introducing the subject of this paper Dr. Childs laid special stress upon the remarkable parallelism in the course of the three epidemics, the evidence showing that the greater part of the primary infection took place before any warning was given through notification; the obscurity and complexity of the problems connected with explosive outbreaks of typhoid fever; our ignorance with regard to these problems ; the exact and exhaustive character of the investigations required for their solution; the need of carrying out these investigations thoroughly from the first, when the medical officer of health and his staff are already more than fully occupied ; and the consequent need of assistant experts in reserve, who should be trained especially for the purpose of investigating these epidemics (as well as endemic typhoid) and ready to come at short notice if applied for by the local medical officer of health.]

A Short Account of THE Lincoln Epidenic. (1021 cases, 120 deaths. December, 1904, to July, 1905.) The city of Lincoln (estimated population about 51,000 ) is built partly upon an elevated plateau of oolitic formation, from which an escarpment descends in steep slopes to the river level some 200 feet below; partly on these sloping sides, and partly on the alluvial beds which encompass the base of the escarpment.

1 An abstract of a paper read before the Incorporated Society of Medical Ufficers of Health on Nov. 10th, 1905 